\title{
Selbstoptimierung 2.0 - das Curriculum Vitae im digitalen Zeitalter
}

\begin{abstract}
Das Curriculum Vitae, die wohl verbreitetste autobiographische Textsorte, basiert ganz wesentlich auf dem Spannungsverhältnis zwischen Selbstreflexion und Selbstoptimierung. Gerade im digitalen Zeitalter scheint das CV dabei immer weniger als Medium der Introspektion über den eigenen Werdegang zu dienen. Der Aufsatz geht der Frage nach, in welcher Weise social media Plattformen wie LinkedIn und XING, die für die erwerbsbiographische Selbstrepräsentation professionalisierte Formate zur Verfügung stellen, die Entfaltung des unternehmerischen Selbst in der Gegenwart noch weiter vorantreiben.
\end{abstract}

Keywords: Curriculum Vitae; Lebenslauf; Biographie; Autobiographie; LinkedIn; XING; Selbstoptimierung

Ein Tweet machte den Psychologieprofessor Johannes Haushofer weit über die Grenzen seiner akademischen Kreise der University of Princeton bekannt. Er erstellte einen CV of Failure, einen Lebenslauf des Scheiterns, der in Umkehrung des traditionellen Formats seine Misserfolge listet, abgelehnte Bachelor- und PhD-Bewerbungen, versagte Fellowships. Als quasi-Motto geht seiner tabellarischen Auflistung voraus: „Das meiste, was ich versuche, gelingt mir nicht. Aber diese Rückschläge sind meistens unsichtbar, während meine Erfolge sichtbar sind. (Krex 2016)

Freilich, Haushofers CV des Scheiterns mutet im Lichte seiner überaus erfolgreichen akademischen Laufbahn etwas eigentümlich an - Bachelor in Oxford, erste Promotion in Harvard, zweite in Zürich, anschließend eine Anstellung am Massachusetts Institute of Technology (MIT). (Vgl. Simon 2016) Und doch: Die Tatsache, dass Haushofers Anti-CV auf solche Resonanz stieß, ist nicht wenig aufschlussreich - der Wissenschaftler hat in einem späteren Tweet gar erklärt, „dieser verdammte CV“ habe mehr Aufmerksamkeit bekommen als seine ganzen bisherigen Forschungen. Der Umstand reflektiert das gesellschaftliche Unbehagen darüber, dass in einer Gesellschaft, die Leistung $\mathrm{zu}$ einer moralischen Qualität erhebt, Rückschläge, Misserfolge und Brüche aus der Vorstellung eines geglückten Werdegangs zwingendermaßen ausgeschlossen bleiben müssen.

Dem Curriculum Vitae, jener Gattung, die für die meisten Personen das einzige Format autobiographischer Selbstreflexion darstellt, kommt damit in der Frage, welche Bilder vom Bios und von der Biographie in der Gesellschaft kur- 
sieren, keine geringe Relevanz zu. Dem CV liegt ein Verständnis von Biographie zugrunde, das das Leben als Aneinanderreihung einzelner Karriere-Positionen eines erwerbstätigen Subjekts erfasst. (Vgl. Kohli 2017, 497) Die Gattung selbst stellt den ,Autobiographen' damit automatisch vor die Zerreißprobe zwischen biographischer Selbsterkundung und gewissenhafter Selbstoptimierung. (Vgl. Heinrich 2014)

Gerade im digitalen Zeitalter scheint das CV indes immer weniger als Medium der Introspektion über den eigenen Lebenslauf zu taugen. Der Aufsatz wird der Frage nachgehen, in welcher Weise social media Plattformen wie LinkedIn und $X I N G$, die professionalisierte Formate für die erwerbsbiographische Selbstrepräsentation zur Verfügung stellen, die Entfaltung des unternehmerischen Selbst in der Gegenwart noch weiter vorantreiben.

\section{Die Institutionalisierung des Lebenslaufs}

Biographische Ordnungsarbeit ist ein „zeitsensitiver“ und „zeitkonstituierender“ Prozess (Fischer-Rosenthal 2000, 457). Er beruht nicht allein auf der hermeneutischen und ordnenden Arbeit des (Auto-)Biographen, nicht nur auf der Selektion und zumeist chronologischen Verdichtung lebensgeschichtlicher Daten. Die Ordnung des Lebenslaufs ist auch an sozial vorgezeichnete Präskripte gebunden (vgl. Berger und Berger 1983, 66 f.), an institutionalisierte Vorstellungen von Zeit. Die gesellschaftlichen Ausdifferenzierungsprozesse im Übergang von der Vormoderne zur Moderne hätten - so hat die soziologische Lebenslaufforschung verstärkt zu zeigen versucht - zunehmend auch zu differenzierteren Vorstellungen von Chronologie und Alter geführt und so auch die Erfahrung der Individualität des eigenen Lebenslaufs ermöglicht. ${ }^{1}$

In diesem Zusammenhang steht auch die Etablierung des kapitalistischen Erwerbssystems, das zu Beginn des 19. Jahrhunderts zunehmend zur institu-

1 Ein paar historische Befunde seien hier nur stichwortartig genannt: Demographische Veränderungen und eine immer höhere Lebenserwartung haben die Vorhersehbarkeit des Lebenslaufes und die Berechenbarkeit von Chronologie zur Folge. Diese bedingen die Ausdifferenzierung von Lebensphasen (Kindheit, Jugend, etc.), die Konstitution von Altersgrenzen, und führen, wie dies Martin Kohli nennt, zu ,altersgeschichteten Systemen öffentlicher Rechte und Pflichten“. (Kohli 2017, 504-505.) Als prägnantes Momentum einer historischen Entwicklung ist in diesem Zusammenhang der Code Napoléon zu Beginn des 19. Jahrhunderts zu nennen, dessen Zivilrecht auf einem altersgestuften System basiert. Ein gesetzliches Schulalter sowie nach chronologischem Alter ausdifferenzierte/s Vertragsfähigkeit, Wahlrecht, Wehrpflicht und Pensionsalter waren die Folge, die amtliche Registrierung des Geburtsjahres sollte dann Ende des 19. Jahrhunderts folgen. 
tionalisierten Dreiteilung des Lebenslaufs führte: die Vorbereitungsphase: Kindheit und Jugend als Zeit der Erziehung, Bildung und Ausbildung; die Aktivitätsphase: die aktive Erwerbstätigkeit; sowie die Ruhephase: das Pensionsalter, in dem die Leistungsfähigkeit immer eingeschränkter ist. Innerhalb der dreiteiligen Sequenzierung der Lebensphasen, die an spezifische Berechtigungssysteme (wie die Rente im Pensionsalter) gekoppelt sind, wird die Phase der Erwerbstätigkeit dabei immer stärker in den Fokus rücken und mit der Ausdifferenzierung von ,Karriere' selbst wiederum eine immer differenziertere Sequenzierung erfahren. (Vgl. Kohli 1985, 504). ${ }^{2}$

Im Zusammenhang des CVs scheinen an dieser Entwicklung fünf Momente entscheidend: Erstens: Als Folge der immer differenzierteren Chronologisierung des Lebens - so wiederum ein Befund Kohlis - kommt es zur schrittweisen Entwicklung einer „Normalbiographie“ (Kohli 2017, 497). Diese nimmt sich zwar einerseits als „Orientierungshilfe“ für das Leben aus, die Vorstellung eines „normalen“, d.h. standardisierten und homogenisierten Lebenslaufs führt jedoch andererseits zur Rationalisierung und sozialen Kontrolle des biographischen Subjekts: Mit der sozialen Institutionalisierung des Lebenslaufs ist auch die Messbarkeit und Vergleichbarkeit der Lebensverläufe, der „nutzbar-gemachten Zeit“ gegeben - Entwicklung und Leistung wird bemessbar.

Zweitens: Der vormoderne Jahreszyklus, an dem sich ein bäuerliches Erwerbsleben noch orientierte, wird in der Moderne von der Vorstellung eines „Ausfüllens des ganzen Lebens“ (Kohli 1985, 505 ff.) abgelöst - damit sind auch differenziertere Vorstellungen von ,Zukunft“ in die Reflexionen über den Verlauf des Lebens integriert. Die Planbarkeit von Zukunft hat ihren hohen Preis: Affektkontrolle, Selbstdisziplinierung, Lustunterdrückung sind das, was die zunehmende Zielgerichtetheit des Lebens einfordert und abverlangt. Norbert Elias spricht in seiner Analyse des Zivilisationsprozesses in diesem Zusammenhang von „Selbstzwang“ und „Zwang zur Langsicht“ (Elias 1969, 336 ff.). Und Max Weber thematisiert die Problemkonstante in seiner idealtypisch-antagonistischen Charakterisierung der protestantischen Ethik:

Der normale mittelalterlich katholische Laie lebte in ethischer Hinsicht gewissermaßen ,von der Hand in den Mund'. Er erfüllt zunächst gewissenhaft die traditionellen Pflichten. Seine darüber hinausgehenden ,guten Werke‘ aber blieben normalerweise eine nicht notwendig

2 Die lebensgeschichtliche Dreiteilung löst damit weit weniger ausdifferenzierte Vorstellungen von Lebenszyklen ab: etwa jene von Geburt - Hochzeit - Tod (vgl. Schenda 1983, 59) oder die ,annalistischen“ Modelle, die eher den über-individuellen Lauf der Zeit (manchmal explizit jahreszeitliche Entwicklungen) oder historische Ereignisse ins Zentrum setzen, und dabei häufig Gott und eben nicht das individualisierte Subjekt in den Mittelpunkt rücken. 
zusammenhängende, zum wenigsten eine nicht notwendigerweise zu einem Lebenssystem rationalisierte Reihe einzelner Handlungen [...]. Der Gott des Calvinismus verlangt von den Seinigen nicht einzelne ,gute Werke', sondern eine zum System gesteigerte Werkheiligkeit. Die ethische Praxis des Alltagsmenschen wurde so ihrer Plan- und Systemlosigkeit entkleidet und zu einer konsequenten Methode der ganzen Lebensführung ausgestaltet. (Weber 1920, 113 ff.)

Mit dem pietistischen Tagebuch etwa ist den Mitgliedern der protestantischen Kirche der systematische Rahmen zur Selbstreflexion geboten, näherhin über das alltägliche moralische Handeln Buch zu führen. (Vgl. Niggl 1977) Die „systematische Selbstkontrolle“ würde auf lange Sicht jedoch - so Weber - die „Vernichtung der Unbefangenheit des triebhaften Lebensgenusses“ herbeiführen. (Vgl. Kohli 2017, 506)

Drittens: Seine Fixierung auf einen „Normallebenslauf“ lässt beim biographischen Subjekt verstärkt das Bedürfnis nach einer eigenen charakteristischen Individualität entstehen. Dieses antagonistische Spannungsverhältnis zwischen gewöhnlichem und außergewöhnlichem Lebensverlauf ist der Gattung der Biographie von je her fundamental eingeschrieben - die Biographie beschreibt „die Abweichungen eines Individuums von einem Modell oder einem Typus.“ (Fetz 2009, 35) Niederschlag findet das Spannungsverhältnis aber auch im Curriculum Vitae, jenem (sehr häufig) standardisierten Text-Format, das vom Bewerbungskandidaten eine einzigartige Individualität zu präsentieren verlangt. ${ }^{3}$

Viertens: Die (narrativ oder nur tabellarisch hergestellte) Individualität des Subjekts gründet im Curriculum Vitae auf der Konstruktion eines vermarktbaren Selbst. Das CV soll den Beleg dafür liefern, dass sich der Bewerbungskandidat „den Forderungen der wirtschaftlichen Rationalität bisher bruchlos unterzogen hat." (Kohli 1985, 511) Das Textformat dient dabei paradoxerweise nicht nur als Bilanzierungsmedium für das sich bewerbende autobiographische Subjekt (Heinrich 2014), es fungiert vielmehr als Grundlage für die Zukunftskalkulation möglicher Arbeitgeberinnen und Arbeitgeber, ob und inwieweit der Humankapitaleinsatz lohnend sein wird oder nicht. Das CV ist damit geprägt von einer paradoxen zeitlichen Struktur: Obwohl es im allerkonkretesten Sinne das vergangene Leben eines ökonomischen Subjekts erfasst, soll es doch eigentlich darüber Auskunft geben, welche Zukunft diesem potentiell noch bevorsteht. Es

3 Es sind bis in die neueste Zeit hinein außergewöhnliche Lebensverläufe, die auf das Interesse der Biographinnen und Biographen sowie Leserinnen und Leser stoßen: „Größe“, „Biographiewürdigkeit“ und damit verbunden die superiore „Individualität“ wird von Biographinnen und Biographen häufig über die Loslösung von vorgegebenen (normierten) Zeitstrukturen hergestellt. 
bemisst damit das Verhältnis von gelebter und noch verfügbarer Zeit (Kohli 1983, 511). ${ }^{4}$

Das Format des Curriculum Vitae verlangt damit vom Bewerbungskandidaten, ein aus sich herausgelöstes und an den Erfordernissen der Wirtschaft (am Profil des Betriebs) angepasstes Ich zu konstruieren (Heinrich 2014). Im Kontext des Bewerbungsverfahrens erfährt der Kandidat seine Transformation zur Ware. (Vgl. Klopotek 2014, 19) Das CV basiert damit wesenhaft auf dem Prinzip der Vermarktung, der Logik der kalkulierten ,Schönfärberei‘. Brüche im Lebenslauf werden geglättet, Lücken werden zu produktiven Phasen der Selbstreflexion und SelbstFindung umgedeutet, wozu wiederum die Ratgeberliteratur anregt (Heinrich 2014). Anspruch ist der „lückenlose Lebenslauf“ (Kohli 1985, 511).

Fünftens: In dem Prozess der Vermarktung wird auch die außerhalb des konkreten beruflichen Kontextes stehende Privatperson zum Objekt des Interesses. Der Beleg dafür, dass im Rahmen des Curriculum Vitae die Grenze zwischen Berufsleben und Freizeit zunehmend verschwimmt, liefert der Umstand, dass berufsexterne, private Interessen und Hobbys seit Anfang der 1960er Jahre zunehmend in das CV integriert werden. Gerade „sachfremde“ Bildungs- und Ausbildungsaktivitäten im Zusammenhang des lifelong learning (darunter etwa Kurse im Bereich von Sprachen, Sport und mentaler Fitness) führen vor, dass sich die private Person (im Kontext des Kriterienkatalogs der allumfassenden und permanenten Selbstoptimierung) nicht mehr als externalisierte Einheit fassen lässt.

Nicht nur zeitgenössische Bewerbungen zeugen von der „Ausweitung der Zonen der Selbstoptimierung“ (Klopotek 2014, 20); es ist der Arbeitsmarkt, der mit einer ganzen Reihe von Humanisierungs- und Flexibilisierungsprogrammen zur Zufriedenheit der Angestellten und für deren Leistungsbereitschaft die gegenseitige Durchwirkung der beiden Sphären vorantreibt: neue Arbeitsverhältnisse und Arbeitsstrukturen, flache Hierarchien, flexible Arbeitszeitregelungen (fernab der Stechuhr, Home Office), neues Arbeitsethos (Partizipation, Verantwortung, Selbstunternehmertum), dazugehörige Selbst-Techniken, die als Key-Phrases in heutigen CVs nicht fehlen dürfen (Verantwortungsbereitschaft, Entscheidungsfreude, Spontaneität und Teamfähigkeit) (vgl. Klopotek 2014, 21-22) und last but not least innovative Bürokonzepte. Zeitgenössische Extremformen, an denen sich jedoch gewisse Trends ablesen lassen, stellen die Arbeitswelten von TechnologieGiganten im Silicon Valley dar:

4 Wie Tobias Heinrich in seiner Analyse der Dialektik von „Selbstfindung und Selbstzurichtung im Curriculum Vitae“ zeigt, wird von der Ratgeberliteratur empfohlen, CVs nicht nur für konkrete Bewerbungsanlässe zu schreiben, sondern diese immer wieder auch als Format zur Bilanzierung der bisherigen Leistungen zu verwenden. (Vgl. Heinrich, 4) 
Ein polychromes Google-Fahrrad können sich Angestellte überall auf dem Campus holen und abstellen, so dass andere es benutzen können. Mit elektrischen Autos, die an einer Aufladestation stehen, können Mitarbeiter ihre Einkäufe erledigen. Bei Facebook können sie Sushi oder Burritos essen, Gewichte stemmen, sich die Haare schneiden lassen, ihre Kleidung in die Reinigung geben und zum Zahnarzt gehen, alles, ohne den Arbeitsplatz zu verlassen. Währenddessen plant man bei Apple, fast fünf Milliarden Dollar für eine gigantische, undurchdringliche, ringförmige Zentrale auszugeben, die inmitten eines Parks stehen wird, der eigentlich zu Cupertino gehört. Solche autarken und autoreferentiellen Orte erschweren Angestellten sogar zufälligen Kontakt mit der umliegenden Gesellschaft. Die Grenze zwischen privat und öffentlich ist dort verwischt. Unternehmen, die eine derart regressive Umgebung für ihre Mitarbeiter schaffen, erwarten im Gegenzug unbegrenzte Hingabe für die Arbeit. (Harrison 2014)

\section{Das CV in den sozialen Netzwerken}

Das CV stellt einen Extremfall autobiographischen Schreibens dar. Die Textgattung führt nicht nur anschaulich die Entwicklung des Lebenslaufs zu einer sozialen Institution vor Augen, in ihr werden auch - heute mehr denn je - die sozialen Kontrollmechanismen einer sich selbst verwaltenden Gesellschaft augenscheinlich. Gerade dort, wo CVs im World Wide Web öffentlich einsehbar werden - in vielen (vor allem sozial höher bewerteten) Berufssparten stellt die Veröffentlichung eine gängige Praxis dar - nimmt sich die Textform als Instrument der gesellschaftlichen Selbstregulierung aus.

In den letzten zweieinhalb Jahrzehnten haben sich für die Verbreitung erwerbsbiographischer Daten auch eigene Social Media Plattformen erfolgreich etabliert. Das 2002 gegründete soziale Netzwerk LinkedIn, seit 2016 Teil von Microsoft, verwaltet mit weltweit knapp 520 Millionen registrierten Nutzerinnen und Nutzern (vgl. Lippold 2017, 214) in etwa 200 Ländern die größte Menge erwerbsbiographischer Daten. LinkedIn gehöre damit, dem Online-Dienst Alexa nach, zu den 20 meistbesuchten Internetseiten (Stand 2017). Allein im deutschsprachigen Raum sind etwa elf Millionen Menschen auf der Plattform registriert. Die 2002 gegründete Plattform für Geschäftsnetzwerke XING ist mit acht Millionen Nutzerinnen und Nutzern (vgl. Lippold 2017, 214) das Pendant im deutschsprachigen Raum.

Plattformen wie LinkedIn oder XING dienen zur Vernetzung vor allem in beruflichen Kontexten, zur Knüpfung und Pflege geschäftlicher Beziehungen und natürlich dem Self-Branding und Self-Fashioning der User. Die Plattformen werden auch als Präsentationsform für Firmen immer wichtiger. Sie fungieren als entscheidende Bühne für die Unternehmenspräsentation und -vernetzung wie auch als Börse für Talent-Scouting und Personal-Akquise - dies gilt in gesteigerter 
Weise für den explosiv wachsenden Markt an Recruiter- und Head-Hunter-Unternehmen (vgl. Athanas und Graf 2013, 74). Im Business-to-Business-Bereich hätte sich LinkedIn - noch vor Facebook - als beliebtestes Netzwerk etabliert.

Das Profil der User enthält in tabellarisch-chronologischer Form Daten zu aktuellen und früheren Arbeitsverhältnissen und Berufserfahrungen sowie zum bisherigen Ausbildungsweg (nicht selten mit einsehbaren Zertifikaten, Diplomen und gar Zeugnissen): Häufig finden sich Auflistungen weiterer Kenntnisse, Kompetenzen, Publikationen sowie persönlicher Interessen.

Was die hier online einsehbaren CVs von der traditionellen, ,analogen` Textform des CVs unterscheidet? Die Plattformen bieten im Sinne des Web 2.0 interaktive bzw. kollaborative Elemente. Sie ermöglichen die Kontaktaufnahme mit anderen Usern, die Vernetzung mit spezifischen Interessensgruppen sowie die für Social Media Plattformen charakteristischen Follower-Aktivitäten (u. a. sharen, liken, kommentieren).

Indem Netzwerke wie LinkedIn und XING professionalisierte Formate zur Veröffentlichung und Verbreitung erwerbsbiographischer Daten bereitstellen - und damit auch die Vergleichbarkeit von Lebensläufen professionalisieren -, haben sie aktiv daran teil, den Leistungsdruck ihrer Nutzerinnen und Nutzer zu erhöhen und deren Leistungsbereitschaft zu steigern.

Zwar wird der Wettbewerb um das Humankapital hier auf offener Bühne (für alle einsehbar) ausgetragen, der Mechanismus der Kontrolle über die Akteurinnen und Akteure, der diesen Wettbewerb letztlich am Laufen hält, wirkt indes immer versteckter. Wesentlich erscheint dabei, dass die öffentliche Einsehbarkeit der individuellen Leistungen eine Kontroll- und Überwachungskonstellation schafft, die ohne die konkrete Disziplinierung einer Ordnungsmacht auskommt. Die gegenseitige Überwachung und Disziplinierung bleibt den erwerbsbiographischen Subjekten selbst überlassen, die zeitgenössische digitale Welt ist von einer Internalisierungsmacht durchwirkt. ${ }^{5}$

5 Die (Selbst-)Disziplinierungsmechanismen, die in der Verbreitungspraxis der Curriculum Vitae-Netzwerke manifest werden, ließen sich mit Blick auf Jeremy Benthams „Panopticon“, den architektonischen Entwurf eines perfekten Gefängnisses, veranschaulichen; Der Entwurf sieht vor, dass von einem Beobachtungsturm im Mittelpunkt einer kreisförmigen Anlage alle unter Beobachtung stehenden Insassen (Gefängnisinsassen oder Fabriksarbeiter) beaufsichtigt werden können. Das Wesentliche des Entwurfs ist dabei, dass der Wärter die Insassen beobachten kann, ohne von den Insassen gesehen zu werden. Für die Insassen bleibt somit unklar, ob sie einer Beobachtung ausgesetzt sind oder nicht. In Foucault Schrift Überwachen und Strafen dient der Entwurf bekanntlich als Denkfigur für das Ordnungsprinzip der westlichen Gesellschaft seit dem 18. Jahrhundert. Benthams Konstruktion ließe sich - auf die Selbstdisziplinierungsgesellschaften des 21. Jahrhunderts übertragen - ohne den Beobachtungsturm im Mittelpunkt der Anlage 
Die Disziplinargesellschaften, die sich zwischen dem 18. und 20. Jahrhundert formierten, hätten die zu disziplinierenden Personengruppen, so Foucault, in spezifischen Einschließungsmilieus geordnet - zu nennen sind hier etwa die Familie, Schule, Kaserne und Fabrik; Extremformen stellen die Klinik und das Gefängnis dar. In den Kontrollgesellschaften, die sich nach Deleuze in den westlichen Länder ab den 1990er Jahren etabliert hätten, sei das Unternehmen an die Stelle der Fabrik getreten, das lebenslange Lernen habe die Schule abgelöst, anstelle von sporadischen Prüfungen (d.h. zeitlich kompakter Disziplinierung mit anschließendem Freizeitausgleich) (vgl. Ernst 2013, 36) institutionalisierten sich kontinuierliche, permanente und vor allem flexibilisierte Kontrollen: „Der Mensch der Disziplinierung war ein diskontinuierlicher Produzent von Energie, während der Mensch der Kontrolle eher wellenhaft ist, in einem kontinuierlichen Strahl, in einer Umlaufbahn.“ (Deleuze 1993, 258) Deleuze erklärt, dass sich in der Kontrollgesellschaft zudem die Heimarbeit zu einem wesentlichen „Produktionsfaktor“ entwickelt hätte (Ernst, 2013, 36), während Heim und Arbeit in der Disziplinargesellschaft noch zwei voneinander getrennte (räumliche) Sphären gewesen seien - er spricht von der „Einführung des Unternehmens auf allen Ebenen des Bildungs- und Ausbildungswesens“ (Deleuze 1993, 261). Das trifft insbesondere auf die digitale Arbeitswelt zu, wo die Produktionsbedingungen vollkommen vom Produktionsort abgekoppelt sind; auch Plattformen wie LinkedIn und XING sind in gleicher Weise von Zuhause wie vom Büro aus zu bespielen.

Andreas Bernard geht nun noch einen Schritt weiter, wenn er mit Rückgriff auf Foucaults Disziplinargesellschaft und das Konzept der Kontrollgesellschaft bei Deleuze erklärt, hinter den Entwicklungen des 21. Jahrhunderts eine neue, „dritte Ausprägung“ zu sehen, „die man Internalisierungsmacht nennen könnte.“ (Bernard 2015) Der Mechanismus ist ein einfacher: Unabhängig von einer tatsächlich stattfindenden „Überwachung“ diszipliniere sich das unter potenzieller Beobachtung stehende Individuum selbst, indem es sein Verhalten den normativen Erwartungen anpasse - so lange, bis es auch das Bewusstsein dafür verloren hat, soziales Handeln auf der Basis systematischer Kontrolle zu vollziehen. ${ }^{6}$

denken. Denn die zeitgenössischen Vergesellschaftungsformen, in denen das digitale Subjekt (mitsamt seinem digitalisierten Lebenslauf) ständig der Gefahr ausgesetzt ist, von anderen beobachtet und beurteilt zu werden, kommt letztlich gänzlich ohne Aufsicht aus.

6 Der Prozess lässt sich mit Felix Klopotek als „Transfer“ beschreiben: „Ein Druck von außen, sich anzupassen der ,Kultur‘ eines Unternehmens, den Gesetzen des Marktes, den Zumutungen der Politik, ein guter Staatsbürger zu sein, die Imperative der Kapitalverwertung werden übersetzt in eine Norm, eine moralische Vorschrift, die ,ich persönlich` zu verwirklichen habe. Man kann folglich von Subjektivierungsprozessen sprechen. [...] Die Leute verinnerlichen aber nicht nur ein Gebot und verhalten sich dann ohne äußeren Zwang, sondern das, was sie verinner- 
Für Richard Sennett zeigen sich exemplarisch im Teamwork die neuen Machtstrukturen: Den Mitarbeiterinnen und Mitarbeitern wird suggeriert, beim Problemlösungsprozess involviert und in der Arbeitsorganisation gleichberechtigt zu sein, de facto werden die Macht-, Kontroll- und Evaluierungsprozesse ins Interne der Bürogemeinschaft verlagert. ${ }^{7}$ Macht und Kontrolle sind bis ins Unsichtbare hinein internalisiert: „Flexible capitalism has blocked the straight roadway of career“. Der Kapitalismus suggeriere, „flexibility gives people more freedom to shape their lives. In fact the new order substitutes new controls rather than simple abolishing the rules of the past - and these new controls are also hard to understand.“ (Sennett 1999, 9-10)

Da, wo Selbstoptimierung beinah widerspruchslos zum Teil des alltäglichen sozialen Handelns und damit zur moralischen Kategorie der Selbstbeschreibung einer Gesellschaft wird, ist sie als gesellschaftliche Praxis internalisiert. Das zeigt sich auch im Kontext des digitalen CVs. Während das traditionelle CV ausschließlich konkreten Bewerbungszwecken diente, fungiert es in der digitalen Gegenwart als permanente Visitenkarte des unternehmerischen Selbst. Die Arbeit am Curriculum Vitae wird damit zu einem Teil der Arbeit selbst. Als Bestandteil der Social Media Aktivitäten (von Firmen, Institutionen, Selbstständigen) gehört die SelbstVermarktung zum Anforderungsprofil in der heutigen Berufswelt. Der Erfolg von Unternehmen vieler kommunikationsintensiver Branchen hängt in Zeiten von LinkedIn und XING zu einem nicht unwesentlichen Teil davon ab, ob die Angestellten als „freiwillige Multiplikatoren“ in Social Networks reüssieren (vgl. Athanas und Graf 2013, 123), davon also, ob sie als erwerbsbiographische Subjekte (mit ihrem Lebenslauf) vermarktbar sind. Nicht die Qualität des eigenen CVs allein wird damit zum Bewertungskriterium, sondern vielmehr die individuelle Fähigkeit, das CV - die eigene Leistungsbilanz - in der medialen Öffentlichkeit gezielt zu divulgieren. Die Follower-Anzahl der Nutzerinnen und Nutzer von XING und LinkedIn wird damit in Berufs- und Freizeitkontexten zu einem wesentlichen Bewertungskriterium des Erfolgs des unternehmerischen Selbst.

Die neue, prekäre Qualität der digitalen Internalisierungsmacht ist - wie gesagt - ihr Verhüllungsmechanismus: Im Kontext des interaktiven Kommunikationsrahmens der social networks, der sich aus spielerischen Akten des Likens,

lichen, eröffnet ihnen einen Zugang zum Handeln, wird mit dem Handeln, der Praxis, immer wieder abgeglichen. Die Verinnerlichung muss verwirklicht werden, und dieses ,Verwirklichen“ verweist wieder auf ein Außen.“ (Klopotek 2016, 12-13)

7 Der Selbstbetrug bestehe darin, ,,von dem Fehlen einer Hierarchie innerhalb der Bürogemeinschaft auf das Ende der Hierarchien in der gesamtgesellschaftlichen Arbeitsteilung zu schließen, die Abwesenheit eines Chefs mit der Abwesenheit von Zwang zu verwechseln. Die Hierarchie ergibt sich aus dem Zwang zum Profit“ (Klopotek 2014, 23). 
Sharens und Kommentierens speist - internalisierten Akten einer lustvollen Freizeitbeschäftigung -, scheint die Selbstverlistung und -vermarktung selbst zu einem spielerischen Akt geworden zu sein. Das einzelne Subjekt arbeitet damit selbst an seiner Quantifizierung bereitwillig mit.

Darüber hinaus suggerieren Plattformen wie LinkedIn und XING den Nutzerinnen und Nutzern, Kontrolle hätte sich demokratisiert. Die Plattformen basieren auf einem Referenz- und Bewertungssystem, in dem die Mitglieder als Kontrolleurinnen/Kontrolleure und Kontrollierte, als Bewerterinnen/Bewerter und Bewertete gleichermaßen auftreten. Angaben der Nutzerinnen und Nutzer über ihre Kenntnisse, Kompetenzen, bisherigen Berufserfahrungen werden von anderen Nutzerinnen und Nutzern per Mouse-Click offiziell authentifiziert. Aktiveren Mitgliedern der Plattform bietet sich die Möglichkeit, bisherigen Projektpartnerinnen und -partnern Zeugnisse und Empfehlungsschreiben für die erfolgte Zusammenarbeit - wiederum für andere Nutzerinnen und Nutzer einsehbar - auszustellen. Ein interaktives Empfehlungs-Netzwerk (mit Kontrollfunktion) entsteht.

Ob das traditionelle Curriculum Vitae (des Bewerbungsdossiers) angesichts neuer digitaler CV-Formate ein Ablaufdatum hat, wird sich zeigen. Klar scheint jedoch bereits jetzt, dass lebensgeschichtliche Daten, dort, wo sie in Social Media Masken einspeist und sukzessive von weiteren Variablen der eigenen Leistungsbilanz ergänzt werden, immer weniger als Ausgangspunkt einer Introspektion über das bisherige und zukünftige Leben, über die eigene lebensgeschichtliche Identität dienen können. Das ,Aufschreiben des ganzen Lebens' weicht hier einer tabellarischen Selbst-Quantifizierung. Ein solches Curriculum Vitae hat als Medium der Selbstbespiegelung, als résumé - wie die Bedeutung des im Englischen gebräuchlichen französischen Lehnworts noch vermittelt - nun gänzlich ausgedient.

\section{Literaturverzeichnis}

Athanas, Christoph, und Nele Graf (Hg.). Innovative Talentstrategien - Mit Arbeitshilfen Online: Talente finden, Kompetenzen fördern, Know-How binden. Freiburg: Haufe Lexware, 2013.

Berger, Peter, und Brigitte Berger. Das Unbehagen in der Modernität. Frankfurt/M: Campus, 1975.

Bernard, Andreas. „Die neue Präventionskultur. Wir sind total kontrolliert“. FAZ (28.5.2015). (http://www.faz.net/aktuell/feuilleton/die-neue-praeventionskultur-wir-sind-totalkontrolliert-13607780.html [26.8.2018]).

Bröckling, Ulrich. „Die Arbeit des unternehmerischen Selbst“. Gegenblende 14 (2012): 13-30. Deleuze, Gilles. „Postskriptum über die Kontrollgesellschaften“. Unterhandlungen 1972-1990. Frankfurt/M.: Suhrkamp, 1993. 254-262. 
Distelhorst, Lars. „Die Glühbirne und der Möbelpacker. Über den Begriff ,Leistung‘ als leere Abstraktion." Zonen der Selbstoptimierung. Berichte aus der Leistungsgesellschaft. Hg. Felix Klopotek und Peter Scheiffele. Berlin: Matthes \& Seitz, 2016. 35-53.

Elias, Norbert. Über den Prozeß der Zivilisation. Bern und München: Francke, 1969.

Ernst, Thomas: Literatur und Subversion. Politisches Schreiben in der Gegenwart. Bielefeld: transcript, 2013.

Fetz, Bernhard. „Biographisches Erzählen zwischen Wahrheit und Lüge, Inszenierung und Authentizität“. Handbuch Biographie. Methoden, Traditionen, Theorien. Hg. Christian Klein. Stuttgart: J. B. Metzler, 2009. 54-61.

Foucault, Michel. Überwachen und Strafen. Frankfurt/M.: Suhrkamp, 1977.

Harrison, Robert Pogue: „Kalifornische Ideologie. Verändert die Welt, und macht sie flach!“

FAZ (11.8.2014). (http://www.faz.net/aktuell/feuilleton/kalifornische-ideologie-veraendert-diewelt-und-macht-sie-flach-13092376.html [26.8.2018]).

Heinrich, Tobias. „Selbst(er)findung im Lebenslauf. Plurale Identitäten und narrative

Kohärenz.“ Vortrag im Rahmen des Workshops „Invention and Memory in Biography“ an der Universität Salzburg (19. Mai 2014).

Klopotek, Felix. „On Time Run. Immer unterwegs, niemals ankommen, auf dem Weg durch die Zonen der Selbstoptimierung." Zonen der Selbstoptimierung. Berichte aus der Leistungsgesellschaft. Hg. Felix Klopotek und Peter Scheiffele. Berlin: Matthes \& Seitz, 2016. 9-35.

Kohli, Martin. „Die Institutionalisierung des Lebenslaufs. Historische Befunde und theoretische Argumente.“ Kölner Zeitschrift für Soziologie und Sozialpsychologie 69 (2017): 495-524.

Krex, Alexander. „CV of Failures: Princeton-Professor, 36, gescheitert“. ZEIT Campus (3.5.2016). (https://www.zeit.de/campus/2016-05/cv-of-failures-johannes-haushoferprofessor-princeton-scheitern [26.8.2018]).

Lippold, Dirk. Marktorientierte Unternehmensführung und Digitalisierung: Management im digitalen Wandel. Berlin und Boston: De Gruyter, 2017.

Niggl, Günter. Geschichte der deutschen Autobiographie im 18. Jahrhundert. Stuttgart: Metzler, 1977.

Rosenthal, Gabriele/Fischer-Rosenthal, Wolfram. „Analyse narrativ-biographischer

Interviews”. Qualitative Sozialforschung. Hg. Uwe Flick, Ernst von Kardorff und Ines Steinke. Reinbek: Rowohlt 2000. 456-468.

Schenda, Rudolf. „Bewertungen und Bewältigungen des Alters aufgrund volkskundlicher Materialien “. Gerontologie und Sozialgeschichte. Hg. Conrad von Kondratowitz. Berlin: Deutsches Zentrum für Altersfragen e.V., 1983.

Simon, Felix. „Lebenslauf der Fehlschläge. Schöner Scheitern.“ FAZ (5.5.2016). (http://www. faz.net/aktuell/feuilleton/lebenslauf-der-fehlschlaege-schoener-scheitern-14213919.html [26.8.2018]).

Weber, Max. Gesammelte Aufsätze zur Religionssoziologie. Bd. I. Tübingen: Mohr Siebeck, 1920. 
David Österle ist wissenschaftlicher Mitarbeiter am Institut für Germanistik der Universität Wien. Von 2011 bis 2019 war er wissenschaftlicher Mitarbeiter, zuletzt Stv. Leiter des Ludwig Boltzmann Instituts für Geschichte und Theorie der Biographie. $\mathrm{Zu}$ seinen Forschungsschwerpunkten gehören die Literatur der Jahrhundertwende, Kultur- und Raumtheorie sowie Geschichte und Theorie der Biographie. Seine Dissertation verfasste er über Räume und Gegen-Räume der Moderne im Frühwerk und in der Biographie Hugo von Hofmannsthals; 2019 veröffentlichte er eine Gruppenbiographie zum Jungen Wien („Freunde sind wir ja eigentlich nicht“. Hofmannsthal, Schnitzler und das Junge Wien). 